\title{
Caustics of translation surfaces in Euclidean 3-space
}

\author{
Jingren Chen ${ }^{a}$, Haiming Liu ${ }^{b, *}$, Jiajing Miao ${ }^{b}$ \\ a School of Mathematical Sciences, Harbin Normal University, Harbin, 150025, P. R. China. \\ ${ }^{b}$ School of Mathematical Sciences, Mudanjiang Normal University, Mudanjiang, 157011, P. R. China.
}

Communicated by C. Park

\begin{abstract}
The aim of this paper is to classify the singularities of caustics, which have implications for a wide range of physical applications, of translation surfaces. In addition, we give a particular study on ridge point, sub-parabolic ridge point, and constant curvature line on translation surface and we find that there is no elliptic umblic on translation surface. (C) 2017 All rights reserved.
\end{abstract}

Keywords: Translation surface, caustics, singularity theory.

2010 MSC: 32S25, 53A35.

\section{Introduction}

The study of caustics and wave front evolution has a rich history; it dates back to the early studies of Newton and Huygens, Cayley studied the normal wave front evolution from the triaxial ellipsoid in the middle of the 19th century. The contemporary study of generic wave front and caustic behavior arose in the mid-century via the classification studies of the singularities of functions and mappings. It arose mainly via the efforts of the mathematicians, Whitney, Thom, and Arnold. With several notable exceptions, in particular Berry and Zeldovich, physicists seem to have largely ignored the subject, even though it has implications for a wide range of physical applications; all forms of wave propagation, both classical and quantum mechanical; from geometric optics through to physical optics; intensity distributions in interference and diffraction phenomena, e.g., evaluations of the Fresnel and Airy integrals; gravitational lensing; structure formation in the early universe and in galaxies via density waves; finite size image disruption, Hamilton Jacobi theory; stability problems; thermodynamics; elasticity theory; and states of quantum fields in curved space times $[1,2,8,9,12,21]$. For example, in physical terms the caustic is described as the hypersurface swept by the cusped edges of moving fronts $[1,2,4,5,9,21]$. The propagation of fronts can be described in terms of space-time as a single hypersurface-the union of the momentary fronts belonging to different isochrone hypersurfaces $t=$ const. This hypersurface (for the case of a simple singularity corresponding to a reflection group) is locally diffeomorphic to the discriminant of the

\footnotetext{
*Corresponding author

Email addresses: chenjingren@ruc.edu.cn (Jingren Chen), liuhm468@nenu.edu.cn (Haiming Liu), jiajing0407@126.com (Jiajing Miao)
}

doi:10.22436/jnsa.010.10.16 
reflection group. The cusped edges of the momentary fronts sweep the cusped edge of the discriminant hypersurface. Its projection into physical space along the world lines is the surface in physical space swept out by the cusped edges of the momentary fronts. Thus the generic lines of the mathematical definition of the caustic of a reflection group are the space-time world lines of the physical description. The noncrystallographic Euclidean reflection groups appear in this theory as the description of the propagation of fronts in a manifold with a boundary, for instance in the problem of the fastest bypassing of an obstacle bounded by a hypersurface in ordinary Euclidean space.

A common situation where caustics are visible is when light shines on a drinking glass. The glass casts a shadow, but also produces a curved region of bright light. In ideal circumstances (including perfectly parallel rays, as if from a point source at infinity), a nephroid-shaped patch of light can be produced. Rippling caustics are commonly formed when light shines through waves on a body of water. Another familiar caustic is the rainbow. Scattering of light by raindrops causes different wavelengths of light to be refracted into arcs of differing radius, producing the bow.

A surface $M$ in the Euclidean space is called a translation surface if it is given by an immersion

$$
X: U \rightarrow \mathbb{E}^{3}:(s ; t) \mapsto(s, t, f(s)+g(t)),
$$

where $z(s, t)=f(s)+g(t)$ and $f$ and $g$ are smooth functions on some interval of $\mathbb{R}$. Liu and his coauthors gave the classification of the translation surfaces with constant mean curvature or constant Gauss curvature in 3-dimensional Euclidean space $\mathbb{E}^{3}$ and 3-dimensional Minkowski space $\mathbb{E}_{1}^{3}$. They also classified minimal affine translation surfaces in three dimensional Euclidean space, see [15-17, 23]. In [7], Bekkar and Senoussi studied the translation surfaces in the 3-dimensional space satisfying $\Delta^{\mathrm{III}} r_{i}=\mu_{i} r_{i}$. In [20], Verstraelen et al. studied the minimal translation surfaces in Euclidean space. Yoon studied the Gauss map of translation surfaces in Minkowski 3-space, see [22]. Goemans and Woestyne studied translation surfaces with vanishing second Gaussian curvature in Euclidean and Minkowski 3-space, see [11]. When the ambient is the space $\mathrm{Sol}^{3}$, translation surfaces whose mean curvature vanishes were classified, see [18]. Their works focus on differential equations as the basic tool.

The importance of the study of caustics of translation surfaces and its presence in the mathematical theories and physics are clear. However, to the best of the authors' knowledge, the properties of caustics of translation surfaces have not been considered in the literature. Thus the current study hopes to serve such a need. In this paper, we define the notions of caustics, the pedal surface and the cylindrical pedal of translation surface and we introduce some properties on Gauss mapping of translation surface in Proposition 2.2. Additionally, we give a particular study on ridge point, sub-parabolic ridge point, and constant curvature line on translation surface in Proposition 3.1, Proposition 4.1, and Proposition 4.2, respectively. Moreover, we find that there is only possibly right angled hyperbolic umblic on translation surface (c.f. Proposition 3.2) and we give a classification on the singularity types of caustics of translation surfaces (c.f. Theorem 5.1).

The rest of this paper is organized as follows. Firstly, we introduce some properties of Gauss mapping of translation surface. Then, we give a particular study on ridge point, sub-parabolic ridge point, and constant curvature line on translation surface. Afterwards, we classify the singularity types of caustics of translation surfaces.

\section{Properties of Gauss mapping of translation surface}

In this section, we get some properties on Gauss mapping of translation surfaces as a particular application of the theory in $[3,6,10,13,14,19]$. The main results are contained in Proposition 2.2.

A translation surface in Euclidean 3-space is a surface that is parameterized by

$$
X(s, t)=(s, t, f(s)+g(t)) .
$$

We use a special Monge form in which, at $s=t=0, f(0)=g(0)=f^{\prime}(0)=g^{\prime}(0)=0$. Then at $s=t=0$, the tangent plane to the surface at the origin is the $s, t$-plane. In that case the equation of the surface can 
be written in the following form

$$
f(s)+g(t)=\frac{1}{2}\left(k_{1}(0) s^{2}+k_{2}(0) t^{2}\right)+\sum_{i \geqslant 3} \frac{f^{(i)}(0)}{i !} s^{i}+\sum_{j \geqslant 3} \frac{g^{(j)}(0)}{j !} t^{j} .
$$

Note that there is no st-term in above equation. This indicates that the principal directions at the origin become the directions $(1,0,0),(0,1,0)$ and $k_{1}(0), k_{2}(0)$ are the principal curvatures. For a translation surface, we can compute that the unit normal vector field

$$
\mathbf{n}(s, t)=\frac{1}{\sqrt{1+\left(f^{\prime}(s)\right)^{2}+\left(g^{\prime}(t)\right)^{2}}}\left(-f^{\prime}(s),-g^{\prime}(t), 1\right) .
$$

The map $\mathbf{N}: U \rightarrow S^{2}(1)$ defined by $\mathbf{N}(s, t)=\mathbf{n}(s, t)$ is called the Gauss map of $M=\mathbf{X}(\mathrm{U})$. By the Weingarten formula, the Gauss-Kronecker curvature is given by

$$
K(s, t)=\frac{f^{\prime \prime}(s) g^{\prime \prime}(t)}{\left(1+\left(f^{\prime}(s)\right)^{2}+\left(g^{\prime}(t)\right)^{2}\right)^{2}} .
$$

If the principal curvature $\kappa_{i}(s, t) \neq 0$, then we can define the caustic of $\mathbf{X}(s, t)=M$ by

$$
\mathrm{C}_{\mathrm{M}_{i}}(\mathrm{~s}, \mathrm{t})=\left\{\mathbf{X}(\mathrm{s}, \mathrm{t})+\frac{1}{\mathrm{~K}_{\mathrm{i}}(\mathrm{s}, \mathrm{t})} \mathbf{n}(\mathrm{s}, \mathrm{t}) \mid(\mathrm{s}, \mathrm{t}) \in \mathrm{U}\right\}
$$

We also define the parallel surface of the translation surface at time $u$ by

$$
\mathbf{X}^{\mathfrak{u}}(s, \mathrm{t})=\mathbf{X}(s, \mathrm{t})+\mathrm{un}(\mathrm{s}, \mathrm{t}) .
$$

It is well-known that the caustic is one of parallel surfaces when $u=\frac{1}{\kappa_{i}(s, t)}$. We define the pedal surface of $M=\mathbf{X}(\mathrm{U})$ by

$$
\mathrm{Pe}_{M}: \mathrm{U} \rightarrow \mathbb{R}^{\mathrm{n}} ; \mathrm{Pe}_{M}(\mathrm{~s}, \mathrm{t})=\langle\mathbf{X}(\mathrm{s}, \mathrm{t}), \mathbf{n}(s, \mathrm{t})\rangle \mathbf{n}(\mathrm{s}, \mathrm{t}) .
$$

The parametrization of component of $\mathrm{Pe}_{M}$ is

$$
P e_{M}=\frac{s f^{\prime}+t g^{\prime}-f-g}{1+\left(f^{\prime}(s)\right)^{2}+\left(g^{\prime}(t)\right)^{2}}\left(f^{\prime}, g^{\prime},-1\right)
$$

Concerning on the pedal surface in $\mathbb{R}^{3}$, we define the cylindrical pedal of $M=\mathbf{X}(\mathrm{U})$ by

$$
\mathrm{CPe}_{\mathrm{M}}: \mathrm{U} \rightarrow \mathrm{S}^{2} \times \mathbb{R} ; \mathrm{CPe}_{\mathrm{M}}(\mathrm{s}, \mathrm{t})=(\mathbf{n}(\mathrm{s}, \mathrm{t}),\langle\mathbf{X}(\mathrm{s}, \mathrm{t}), \mathbf{n}(\mathrm{s}, \mathrm{t})\rangle) .
$$

The parametrization of component of $\mathrm{CPe}_{M}$ is

$$
\mathrm{CP} e_{M}=\frac{1}{1+\left(f^{\prime}(s)\right)^{2}+\left(g^{\prime}(t)\right)^{2}}\left(-f^{\prime},-g^{\prime}, 1,-s f^{\prime}-t g^{\prime}+f+g\right) .
$$

We now define two kinds of functions families in order to describe the Gauss map, the caustics and the pedal surface of a translation surface in $\mathbb{R}^{3}$ as

$$
\mathrm{H}: \mathrm{U} \times \mathrm{S}^{2} \rightarrow \mathbb{R} \text { by } \mathrm{H}(\mathrm{u}, \mathbf{v})=\langle\mathrm{X}(\mathrm{u}), \mathbf{v}\rangle
$$

and

$$
\mathrm{D}: \mathrm{U} \times \mathbb{R}^{3} \rightarrow \mathbb{R} \text { by } \mathrm{D}(\mathrm{u}, \mathbf{x})=\|\mathbf{X}(\mathbf{u})-\mathbf{x}\|^{2} .
$$

We call $\mathrm{H}$ a height function and $\mathrm{D}$ distance squared function on $\mathrm{M}=\mathbf{X}(\mathrm{U})$. We denote that $h_{v}(u)=H(u, v)$ and $d_{x}(u)=D(u, x)$. The following proposition follows from direct calculations. 
Proposition 2.1. Let $\mathbf{X}: \mathrm{U} \rightarrow \mathbb{R}^{3}$ be a translation surface in Euclidean 3-space parameterized by $\mathbf{X}(\mathrm{s}, \mathrm{t})=$ $(s, t, f(s)+g(t))$, then we have

(1) $\left(\partial h_{v} / \partial u_{i}\right)(u)=0(i=1, \ldots, n)$ if and only if $v= \pm \frac{1}{L(s, t)}\left(f^{\prime}(s), g^{\prime}(t),-1\right)$;

(2) $\left(\partial \mathrm{d}_{v} / \partial \mathrm{u}_{\mathfrak{i}}\right)(\mathrm{u})=0(\mathfrak{i}=1, \ldots, n)$ if and only if there exist a real number $\lambda$ such that

$$
\mathbf{v}=\frac{1}{\mathrm{~L}(\mathrm{~s}, \mathrm{t})}\left(\mathrm{L}(\mathrm{s}, \mathrm{t}) \mathrm{s}+\lambda \mathrm{f}^{\prime}(\mathrm{s}), \mathrm{L}(\mathrm{s}, \mathrm{t}) \mathrm{t}+\lambda \mathrm{g}^{\prime}(\mathrm{t}), \mathrm{L}(\mathrm{s}, \mathrm{t})(\mathrm{f}(\mathrm{s})+\mathrm{g}(\mathrm{t}))-\lambda\right),
$$

where $\mathrm{L}(\mathrm{s}, \mathrm{t})=\sqrt{1+\left(\mathrm{f}^{\prime}(\mathrm{s})\right)^{2}+\left(\mathrm{g}^{\prime}(\mathrm{t})\right)^{2}}$.

By Proposition 2.1, we can detect both the catastrophe sets of $\mathrm{H}$ and $\mathrm{D}$ as follows:

$$
\begin{aligned}
& \mathfrak{C}(H)=\left\{(u, v) \in U \times S^{2} \mid \mathbf{v}= \pm \frac{1}{L(s, t)}\left(f^{\prime}(s), g^{\prime}(t),-1\right)\right\}, \\
& \mathfrak{C}(D)=\left\{(u, v) \in U \times \mathbb{R}^{3} \mid \mathbf{v}=\frac{1}{L(s, t)}\left(L s+\lambda f^{\prime}(s), L t+\lambda g^{\prime}(t), L(f(s)+g(t))-\lambda\right)\right\} .
\end{aligned}
$$

We can get that the bifurcation set of $\mathrm{D}$ is

$$
\mathfrak{B}_{\mathrm{D}}=\left\{\mathrm{C}_{M_{i}}(s, \mathrm{t}) \mid(s, \mathrm{t}) \in \mathrm{U}\right\}
$$

In the following, we give another geometric interpretation of caustics from the Lagrangian view point. We denote by

$$
\Sigma(\mathrm{D})=\left\{\xi=((u, v), \mathbf{v}) \in \mathrm{U} \times \mathbb{R}^{3} \mid \mathrm{D}_{\mathfrak{u}}(\xi)=\mathrm{D}_{v}(\xi)=0\right\} .
$$

We write $\mathbf{v}=\left(v_{1}, v_{2}, v_{3}\right)$ and denote by $\mathrm{T}^{*} \mathbb{R}^{3}$ the cotangent bundle of $\mathbb{R}^{3}$ endowed with the canonical symplectic structure. Then the map $L(D): \sum(D) \rightarrow T^{*} \mathbb{R}^{3}$, given by

$$
\mathrm{L}(\mathrm{D})((u, v), \mathbf{v})=\left(\mathbf{v},\left(\frac{\partial \mathrm{D}}{\partial v_{1}}((u, v), \mathbf{v}), \frac{\partial \mathrm{D}}{\partial v_{2}}((u, v), \mathbf{v}), \frac{\partial \mathrm{D}}{\partial v_{3}}((u, v), \mathbf{v})\right)\right),
$$

is a Lagrangian immersion. So the map $\pi \circ \mathrm{L}(\mathrm{D}): \Sigma(\mathrm{D}) \rightarrow \mathbb{R}^{3}$ given by $((u, v), \mathbf{v}) \rightarrow \mathbf{v}$ is a Lagrangian map. The caustic $C_{M_{i}}$ of $M$, which is the set of critical values of $\pi \circ L(D)$, is precisely the local stratum of the bifurcation set of the family $D$, i.e., $\mathfrak{B}_{\mathrm{D}}$.

In the terminology of Whitney, the Gauss mapping $\mathrm{N}$ is good if the gradient of Gauss curvature is never zero on the parabolic set. If Gauss map is good, then the parabolic set is a smooth curve $((x(t), y(t))$ and the image $N(t)$ of this curve under the Gauss map is singular precisely when $N^{\prime}(t)=0$. If $N(t)$ is good, then $N$ is excellent if $N^{\prime}(t)=0$ implies $N^{\prime \prime}(t) \neq 0$. Finally, if $N$ is excellent, then $N$ is in general position if the image of $N(t)$ has no triple points or self tangencies, and no cusp point of $N(t)$ coincides with another image point of $N(t)[6]$. We can get the following proposition.

Proposition 2.2. Let $\mathbf{X}: \mathrm{U} \rightarrow \mathbb{R}^{3}$ be a translation surface in Euclidean 3-space parameterized by $\mathbf{X}(\mathrm{s}, \mathrm{t})=$ $(s, t, f(s)+g(t))$. For any $p=\mathbf{X}(s, t)$, we have the following assertions.

(1) The parabolic set of translation surface is $\Sigma=\left\{(\mathrm{s}, \mathrm{t}) \mid \mathrm{f}^{\prime \prime}(\mathrm{s}) \mathrm{g}^{\prime \prime}(\mathrm{t})=0\right\}$ and the Gauss map of translation surface is good if $\mathrm{f}^{(3)}(\mathrm{s}) \mathrm{g}^{\prime \prime}(\mathrm{t}) \neq 0$ or $\mathrm{f}^{\prime \prime}(\mathrm{s}) \mathrm{g}^{(3)}(\mathrm{t}) \neq 0$. Under this condition, the parabolic set is a smooth curve and the Gauss map of translation surface is excellent. Thus the Gauss map is stable with a simple folded along the parabolic curve.

(2) $\mathrm{p}$ is a flat point if and only if $\mathrm{f}^{\prime \prime}(\mathrm{s})=0$ and $\mathrm{g}^{\prime \prime}(\mathrm{t})=0$. Under this condition, the Gauss map of translation surface is not a good map and it is unstable.

Proof.

(1) It is easy to get that the Gauss map of translation surface is given by

$$
\mathbf{N}(s, t)=\frac{1}{\sqrt{1+\left(f^{\prime}(s)\right)^{2}+\left(g^{\prime}(t)\right)^{2}}}\left(-f^{\prime}(s),-g^{\prime}(t), 1\right) .
$$

We can study the singularities of the Gauss mapping more easily in this case by projecting centrally 
from the origin to the plane $z=1$ to get $\left(-f^{\prime}(s),-g^{\prime}(t), 1\right)$. We then project to the $(s, t)$ plane to get the composed mapping $\widetilde{\mathbf{N}}(s, t)=\left(-f^{\prime}(s),-g^{\prime}(t)\right)$. Since the image of $\mathbf{N}$ is contained in the hemisphere, and central projection is a diffeomorphism from the upper hemisphere to the plane $z=1$, the modified Gauss mapping $\widetilde{\mathbf{N}}(s, t)$ will have the same singularities as $\mathbf{N}(s, t)$. In particular $\widetilde{\mathbf{N}}(s, t)$ is singular precisely when its Jacobi matrix has rank less than two, i.e., when the discriminant $\Delta=-f^{\prime \prime}(s) g^{\prime \prime}(t)$ is zero. This indicates that the parabolic set of translation surface is $\Sigma=\left\{(s, t) \mid f^{\prime \prime}(s) g^{\prime \prime}(t)=0\right\}$. Furthermore, $\operatorname{grad}(\Delta)=\left(f^{(3)}(s) g^{\prime \prime}(t), f^{\prime \prime}(s) g^{(3)}(t)\right)$. So $\widetilde{\mathbf{N}}(s, t)$ is $\operatorname{good}$ if $f^{(3)}(s) g^{\prime \prime}(t) \neq 0$ or $f^{\prime \prime}(s) g^{(3)}(t) \neq 0$. Under this condition, we suppose that the parabolic curve is parametrized by $P(t)=(s(t), t)$. The modified Gauss mapping restricted to the parabolic curve is $\widetilde{\mathbf{N}}(\mathrm{t})=\left(-\mathrm{f}^{\prime}(\mathrm{s}(\mathrm{t})),-\mathrm{g}^{\prime}(\mathrm{t})\right)$, with $\widetilde{\mathbf{N}}^{\prime}(\mathrm{t})=\left(-\mathrm{f}^{\prime \prime}(\mathrm{s}) \frac{\mathrm{ds}}{\mathrm{d} t},-\mathrm{g}^{\prime \prime}(\mathrm{t})\right)$. For the case that $f^{\prime \prime}(s)=0$ and $f^{(3)}(s) g^{\prime \prime}(t) \neq 0$, we have that $\widetilde{\mathbf{N}}^{\prime}(t) \neq 0$, so $\widetilde{\mathbf{N}}$ is excellent. Thus the Gauss map is stable, with a simple fold along the parabolic curve. Other case follows from the same computation, so we omit it.

(2) It is easy to get that the Hessian of height function is

$$
\mathcal{H}\left(h_{v}(s, t)\right)=\left(\begin{array}{cc}
\frac{f^{\prime \prime}(s)}{\sqrt{1+\left(f^{\prime}(s)\right)^{2}+\left(g^{\prime}(t)\right)^{2}}} & 0 \\
0 & \frac{g^{\prime \prime}(t)}{\sqrt{1+\left(f^{\prime}(s)\right)^{2}+\left(g^{\prime}(t)\right)^{2}}}
\end{array}\right) .
$$

By the claim (b) of [19, Proposition 3.2], which states that $\mathrm{p}$ is a flat point if and only if $\operatorname{rank}\left(\mathcal{H}\left(\mathrm{h}_{v}(\mathrm{~s}, \mathrm{t})\right)\right)=$ 0 , we get that this is equivalent to $f^{\prime \prime}(s)=0$ and $g^{\prime \prime}(t)=0$.

As applications of Proposition 2.2, we give two examples in the following.

Example 2.3. Consider the shoe surface

$$
X(s, t)=\left(s, t, \frac{1}{3} s^{3}-\frac{1}{2} t^{2}\right)
$$

The parabolic set of this translation surface is $\Sigma=\{(s, t) \mid s=0\}$. Since $f^{(3)}(s) g^{\prime \prime}(t)=-2 \neq 0$, we get that its Gauss map is excellent. Thus, the Gauss map is stable with a simple folded along the parabolic curve $\mathrm{s}=0$.

Example 2.4. Consider the surface

$$
X(s, t)=\left(s, t, \frac{1}{4} s^{4}-\frac{1}{2} t^{2}\right)
$$

The parabolic set of this translation surface is $\Sigma=\{(s, t) \mid s=0\}$. Since $f^{(3)}(0) g^{\prime \prime}(t)=0$ and $f^{\prime \prime}(0) g^{(3)}(t)=$ 0 , we get that its Gauss map is not good.

We also define a family of functions

$$
\widetilde{\mathrm{H}}: \mathrm{U} \times \mathrm{S}^{2} \times \mathbb{R} \rightarrow \mathbb{R} \text { by } \widetilde{\mathrm{H}}(\mathrm{u}, \mathbf{v}, \mathrm{r})=\langle\mathbf{X}(\mathrm{u}), \mathbf{v}\rangle-\mathrm{r} .
$$

We call it the extended height function of $M=\mathbf{X}(\mathrm{U})$. It is easy to get that the catastrophe set of $\widetilde{\mathrm{H}}$ is

$$
\mathcal{D}_{\widetilde{H}}=\left\{ \pm \frac{1}{1+\left(f^{\prime}(s)\right)^{2}+\left(g^{\prime}(t)\right)^{2}}\left(-f^{\prime},-g^{\prime}, 1,-s f^{\prime}-t g^{\prime}+f+g\right) \mid u \in u\right\}
$$

Since the singularities of the pedal and the cylindrical pedal of a hypersurface are diffeomorphic. Although the notion of pedals are classically given, we consider the cylindrical pedal instead of the pedal of $M=\mathbf{X}(\mathrm{U})$.

Proposition 2.5. Both of the distance squared function $\mathrm{D}: \mathrm{U} \times \mathbb{R}^{\mathrm{n}} \rightarrow \mathbb{R}$ and the height function $\mathrm{H}: \mathrm{U} \times \mathrm{S}^{\mathrm{n}} \rightarrow \mathbb{R}$ of $\mathrm{M}=\mathbf{X}(\mathrm{U})$ are Morse families of functions. Especially, the extended height function $\widetilde{\mathrm{H}}: \mathrm{U} \times\left(\mathrm{S}^{\mathrm{n}} \times \mathbb{R}\right) \rightarrow \mathbb{R}$ on $\mathbf{M}=\mathbf{X}(\mathrm{U})$ is also a Morse family. 


\section{Umbilic points, ridge points and sub-parabolic points on translation surfaces}

In this section we get some properties on umbilic, ridge, and sub-parabolic points of translation surfaces. The main results are contained in Propositions 3.1 and 3.2.

At a point on the surface where two principal curvatures are distinct, there are two principal vectors and these vectors are mutually orthogonal. These principal vectors are often colored (blue or red) to distinguish between the two vectors. We assume that $\mathbf{v}_{1}$ is the blue principal vector and $\mathbf{v}_{2}$ is the red principal vector corresponding to principal curvatures $k_{1}, k_{2}$. Suppose that $\mathbf{X}(p)$ is not an umbilic of a regular translation surface $\mathbf{X}(s, t)$. We say that the point $\mathbf{X}(p)$ is a ridge point relative to $\mathbf{v}_{\boldsymbol{i}}$ ("blue" ridge point for $i=1$, "red" for $i=2)$ if $\nabla_{\mathbf{v}_{i}} k_{i}(p)=0$, where $\nabla_{\mathbf{v}_{i}} k_{i}$ is the directional derivative of $k_{i}$ in $\mathbf{v}_{i}$. Moreover, $\mathbf{X}(p)$ is a $k$-th order ridge point relative to $\mathbf{v}_{i}$ if $\nabla_{\mathbf{v}_{i}}^{m_{i}} k_{i}(p)=0,(1 \leqslant m \leqslant k)$ and $\nabla_{\mathbf{v}_{i}}^{k+1} k_{i}(p) \neq 0$. The set of ridge points is called a ridge line or ridges.

Proposition 3.1. Suppose that a translation surface $\mathbf{X}(\mathrm{s}, \mathrm{t})$ is given in Monge form as in (2.1), and that the origin is not an umbilic.

(1) The origin is a first order blue ridge point if and only if

$$
f^{(3)}(0)=0 \text { and }\left(f^{(4)}(0)-3 k_{1}^{3}(0)\right) \neq 0 .
$$

(2) The origin is a second order blue ridge point if and only if

$$
f^{(3)}(0)=\left(f^{(4)}(0)-3 k_{1}^{3}(0)\right)=0 \text { and } f^{(5)}(0) \neq 0 .
$$

(3) Suppose that the origin is a blue ridge point. Then the blue ridge line has a singular point at the origin if and only if

$$
f^{(4)}(0)-3 k_{1}^{3}(0)=0 .
$$

(4) If the origin is a first order blue ridge point, then it is a red sub-parabolic point.

Proof. We remark that the principal curvatures at the origin are $k_{1}, k_{2}\left(k_{1} \neq k_{2}\right)$ with corresponding principal vectors $\mathbf{v}_{1}=(1,0), \mathbf{v}_{2}=(0,1)$. The principal curvature $k_{1}(s, t)$ is expressed as

$$
k_{1}(s, t)=k_{1}+f^{(3)}(0) s+\frac{1}{2}\left[\left(f^{(4)}(0)-3 k_{1}^{3}(0)\right) s^{2}-k_{1}(0) k_{2}^{2}(0) t^{2}\right]+O(s, t)^{3}
$$

and we have

$$
\frac{\partial^{3} k_{1}}{\partial s^{3}}=\frac{f^{(5)}(0)-18 f^{(3)}(0) k_{1}^{2}(0)}{6}
$$

Let $\left(\xi_{1}, \zeta_{1}\right)$ be the eigenvector of weingarten map with the eigenvalue $k_{1}$. Selection of the vector $\left(\xi_{1}, \zeta_{1}\right)$ in order for the tangent vector $\xi_{1} \boldsymbol{X}_{s}+\zeta_{1} \boldsymbol{X}_{\mathrm{t}}$ to be of the unit length shows that the principal vector $\mathbf{v}_{1}$ is expressed as

$$
\mathbf{v}_{1}(s, t)=\left(1+O(s, t)^{2}\right) \frac{\partial}{\partial s}+\left(O(s, t)^{2}\right) \frac{\partial}{\partial t},
$$

and that

$$
\frac{\partial^{2} \zeta_{1}}{\partial s^{2}}(0,0)=0
$$

Therefore, we have

$$
\nabla_{\mathbf{v}_{1}} k_{1}(0,0)=\frac{\partial k_{1}}{\partial s}(0,0)=f^{(3)}(0), \quad \nabla_{\mathbf{v}_{1}}^{2} k_{1}(0,0)=\frac{\partial^{2} k_{1}}{\partial s^{2}}(0,0)+\frac{\partial k_{1}}{\partial t} \frac{\partial \zeta_{1}}{\partial s}(0,0)=f^{(4)}(0)-3 k_{1}^{3}(0,0) .
$$

Moreover, when $\nabla_{\mathbf{v}_{1}} k_{1}(0,0)=\nabla_{\mathbf{v}_{1}}^{2} k_{1}(0,0)=0$, we obtain

$$
\nabla_{\mathbf{v}_{1}}^{3} k_{1}(0,0)=f^{(5)}(0) .
$$


These equations imply the assertions (1) and (2). It follows from (3.1) and (3.2) that the equation of the blue ridge line through the origin is expressed as

$$
\left.\left(f^{(4)}(0)-3 k_{1}^{3}(0,0)\right)\left(k_{1}(0,0)-k_{2}(0,0)\right)\right) s+\cdots .
$$

This equation implies the assertion (3).

Since the principal vectors $\mathbf{v}_{1}$ and $\mathbf{v}_{2}$ are orthogonal, it follows from (3.2) that the principal vector $\mathbf{v}_{2}$ is expressed in the following form:

$$
\mathbf{v}_{2}(s, t)=\left(O(s, t)^{2}\right) \frac{\partial}{\partial s}+\left(1+O(s, t)^{2}\right) \frac{\partial}{\partial t},
$$

the directional derivative $\nabla_{\mathbf{v}_{2}} \mathrm{k}_{1}(\mathrm{~s}, \mathrm{t})$ is given by

$$
\nabla_{\mathbf{v}_{2}} k_{1}(s, t)=-k_{1}(0,0) k_{2}^{2}(0,0) t+O(s, t)^{2} .
$$

This equation implies $\nabla_{\mathbf{v}_{2}} k_{1}(0,0)=0$, so it follows the assertion (4).

We can deduce from (3.4) that the equation of the red sub-parabolic line through the origin has the form

$$
k_{1}(0,0) k_{2}^{2}(0,0)\left(k_{1}(0,0)-k_{2}(0,0)\right) t+\cdots=0 .
$$

Umbilics of a regular surface are points where the two principal curvatures coincide. form

Suppose that the origin is an umbilic of a translation surface $\mathbf{X}(\mathrm{s}, \mathrm{t})$, and that $\mathbf{X}(\mathrm{s}, \mathrm{t})$ is given in Monge

$$
\mathbf{X}(s, t)=(s, t, f(s)+g(t)), f(s)+g(t)=\frac{k(0)}{2}\left(s^{2}+t^{2}\right)+\sum_{i \geqslant 3} \frac{f^{(i)}(0)}{i !} s^{i}+\sum_{j \geqslant 3} \frac{g^{(j)}(0)}{j !} t^{j} .
$$

Here $k$ is the common value for the principal curvatures at the origin. At an umbilic, the cubic part $P_{3}(s, t)$ of $f(s)+g(t)$ in (3.5) determines its type. An umbilic of the surface $\mathbf{X}(s, t)$ is said to be elliptic or hyperbolic if $P_{3}(s, t)=f^{(3)}(0) s^{3}+g^{(3)}(0) t^{3}$ has three real roots or one real root, respectively. Moreover, an umbilic is said to be right-angled if the root directions of the quadratic form which are the determinant of the Hessian matrix of $\mathrm{P}_{3}(\mathrm{~s}, \mathrm{t})$ are mutually orthogonal with respect to the standard scalar product on $\mathbb{R}^{2}$. Such an umbilic necessarily is a hyperbolic umbilic.

Proposition 3.2. There is no elliptic umbilic for translation surface and the origin is possibly only a right-angled hyperbolic umbilic.

Proof. The discriminant of $\mathrm{P}_{3}(\mathrm{~s}, \mathrm{t})=\mathrm{f}^{(3)}(0) \mathrm{s}^{3}+\mathrm{g}^{(3)}(0) \mathrm{t}^{3}$ is given by $\Delta=-\left[\mathrm{f}^{(3)}(0) \mathrm{g}^{(3)}(0)\right]^{2}<0$. Hence, for translation surface, the origin is possibly only a hyperbolic umbilic. Moreover, the determinant of the Hessian matrix of $P_{3}(s, t)$ is given by $\operatorname{det}\left(\operatorname{Hess}_{3}(s, t)\right)=36 f^{(3)}(0) g^{(3)}(0)$ st. If $\operatorname{det}\left(\operatorname{Hess}_{3}(s, t)\right)=$ $36 f^{(3)}(0) g^{(3)}(0) s t=0$, then $s=0$ or $t=0$ which are mutually orthogonal with respect to the standard scalar product on $\mathbb{R}^{2}$. It follows that the origin is a right-angled hyperbolic umbilic of translation surface.

It is shown in [19] that there is one ridge line passing through a hyperbolic umbilic and that ridge line changes its color as it passes through a generic umbilic. It is known that when there is one direction for lines of curvature, there is one sub-parabolic line through the umbilic in the same direction.

\section{Constant curvature lines on translation surfaces}

In this section, we get some properties on constant curvature lines on translation surfaces. The main results are contained in Propositions 4.1 and 4.2. We set $\Sigma_{c}:=\left\{(\mathrm{s}, \mathrm{t}) \mid \mathrm{k}_{\mathrm{i}}(\mathrm{s}, \mathrm{t})=\mathrm{c}\right.$ for some $\left.i\right\}$. We call $\Sigma_{\mathrm{c}}$ the constant principal curvature (CPC) line with the value of $c$. There are two CPC lines $\Sigma_{k_{1}(p)}$ (colored by blue) and $\Sigma_{k_{2}(p)}$ (colored by red) locally through a non-umbilical point $\mathbf{X}(\mathrm{p})$. We recall that a point $p \in U$ is a singular point of the parallel surface $X^{\mathfrak{u}}(p)$ at distance $u$ if and only if $u=\frac{1}{k_{i}(p)}$ for some $i$. This means that the set of singular points of $\mathbf{X}^{\mathfrak{u}}(p)$ is the CPC line $\Sigma_{k_{i}(p)}$. 
Proposition 4.1. Suppose that a translation surface $\mathbf{X}(s, t)$ is given in Monge form as in (2.1), and that the origin is a blue ridge point and red sub-parabolic point. Then the CPC line $\sum_{\mathrm{k}_{1}}$ is locally either an isolated point or two intersecting smooth curves at the origin, if the blue ridge line crosses the red sub-parabolic line at the origin.

Proof. First we remark that $k_{1}(s, t)=k_{1}(0,0)$ is expressed by the equation

$$
f^{(3)}(0) s+\frac{1}{2}\left[\left(f^{(4)}(0)-3 k_{1}^{3}(0)\right) s^{2}-k_{1}(0) k_{2}^{2}(0) t^{2}\right]+O(s, t)^{3}=0 .
$$

It is easy to get $\frac{\partial k_{1}}{\partial s}(0,0)=f^{(3)}(0)=0$ and $\frac{\partial k_{1}}{\partial t}(0,0)=0$. The equations of the blue ridge line (3.3) and the red sub-parabolic line (3.5) reduce to

$$
\left.\left(f^{(4)}(0)-3 k_{1}^{3}(0,0)\right)\left(k_{1}(0,0)-k_{2}(0,0)\right)\right) s+\cdots
$$

and

$$
k_{1}(0,0) k_{2}^{2}(0,0)\left(k_{1}(0,0)-k_{2}(0,0)\right) t+\cdots=0,
$$

respectively. From these equations, the blue ridge line crosses the red sub-parabolic line at the origin if and only if

$$
\left(f^{(4)}(0)-3 k_{1}^{3}(0,0)\right) k_{1}(0,0) k_{2}^{2}(0,0) \neq 0 .
$$

We can get that $\frac{\partial^{2} k_{1}}{\partial s^{2}}(0,0)=f^{(4)}(0)-3 k_{1}^{3}(0,0), \frac{\partial^{2} k_{1}}{\partial s \partial t}(0,0)=0$, and $\frac{\partial^{2} k_{1}}{\partial t^{2}}(0,0)=-k_{1}(0,0) k_{2}^{2}(0,0)$. In addition, the determinant of the Hessian matrix of $k_{1}(s, t)$ at $(0,0)$ is given by

$$
\operatorname{Hess}\left(k_{1}(0,0)\right)=-\left(f^{(4)}(0)-3 k_{1}^{3}(0,0)\right) k_{1}(0,0) k_{2}^{2}(0,0) \text {. }
$$

By the Morse lemma, we complete the proof.

Proposition 4.2. The CPC line $\sum_{k}$ is locally two intersecting smooth curves at a hyperbolic umbilic. The locally two curves change their color as they pass through the hyperbolic umbilic.

Proof. We suppose that the origin is an umbilic of a translation surface. The common principal curvatures are the roots of the principal curvatures equation. In this case, we can express the equation in the form

$$
f^{(3)}(0) g^{(3)}(0) s t+\cdots=0 .
$$

The locus of this equation is the CPC line $\Sigma_{k}$. It is easy to know that there are two intersecting smooth curves at a hyperbolic umbilic from the fact that there is only hyperbolic umbilic point on translation surface.

\section{Classification of singularity points on caustics of translation surfaces}

Now we are ready to classify the singularity types of caustics of translation surface. We obtain the following theorem.

Theorem 5.1. Let $\mathbf{X}(\mathrm{s}, \mathrm{t})$ be a regular translation surface and $\mathrm{C}_{\mathrm{M}_{i}}(\mathrm{~s}, \mathrm{t})$ be its caustic.

(1) If $\mathbf{X}\left(\mathrm{s}_{0}, \mathrm{t}_{0}\right)$ is neither a ridge point relative to the principal direction $\mathbf{v}_{\boldsymbol{i}}$ nor an umbilic $\left(\mathrm{k}_{\mathfrak{i}}\left(\mathrm{s}_{0}, \mathrm{t}_{0}\right) \neq 0\right)$, then the caustic $\mathrm{C}_{\mathrm{M}_{\mathrm{i}}}(\mathrm{s}, \mathrm{t})$ is locally diffeomorphic to the cuspidal edge at $\mathrm{C}_{\mathrm{M}_{\mathrm{i}}}\left(\mathrm{s}_{0}, \mathrm{t}_{0}\right)$.

(2) Suppose that $\mathbf{X}\left(\mathrm{s}_{0}, \mathrm{t}_{0}\right)$ is a ridge point relative to the principal direction $\mathbf{v}_{\boldsymbol{i}}$ and a sub-parabolic point relative to the other principal direction $\mathbf{v}_{j}$, and that $\mathrm{k}_{\mathrm{i}}\left(\mathrm{s}_{0}, \mathrm{t}_{0}\right) \neq 0$. Then the caustic $\mathrm{C}_{\mathrm{M}_{\mathrm{i}}}(\mathrm{s}, \mathrm{t})$ is locally diffeomorphic to the cuspidal lips (resp. cuspidal beaks) at $\mathrm{C}_{M_{i}}\left(\mathrm{~s}_{0}, \mathrm{t}_{0}\right)$ if $\operatorname{det}\left(\operatorname{Hess}_{\left(\mathbf{v}_{1}, \mathbf{v}_{2}\right)} \mathrm{k}_{i}\left(s_{0}, \mathrm{t}_{0}\right)\right)>0$ (resp. $\left.\operatorname{det}\left(\operatorname{Hess}_{\left(\mathbf{v}_{1}, \mathbf{v}_{2}\right)} k_{i}\left(s_{0}, t_{0}\right)\right)<0\right)$ and the order of ridge is one, where Hess $\left(\mathbf{v}_{1}, \mathbf{v}_{2}\right) k_{i}$ is the Hessian matrix of $k_{i}$ with respect to $\mathbf{v}_{1}$ and $\mathbf{v}_{2}$. 
(3) Suppose that $\mathbf{X}\left(\mathrm{s}_{0}, \mathrm{t}_{0}\right)$ is an umbilic which is not a flat umbilic. Then the caustic $\mathrm{C}_{\mathrm{M}_{i}}(\mathrm{~s}, \mathrm{t})$ is locally diffeomorphic to a 3-dimensional $\mathrm{D}_{4}^{+}$singularity at $\mathrm{C}_{\mathrm{M}_{\mathrm{i}}}\left(\mathrm{s}_{0}, \mathrm{t}_{0}\right)$, where the cuspidal edge is a set locally diffeomorphic to the image of a map germ $\mathrm{CE}:\left(\mathbb{R}^{2}, 0\right) \rightarrow\left(\mathbb{R}^{3}, 0\right),(u, v) \mapsto\left(u, v^{2}, v^{3}\right)$, the cuspidal lips is a set locally diffeomorphic to the image of a map germ CLP: $\left(\mathbb{R}^{2}, 0\right) \rightarrow\left(\mathbb{R}^{3}, 0\right),(u, v) \mapsto\left(3 u^{4}+2 u^{2} v^{2}, u^{3}+u v^{2}, v\right)$, the cuspidal beaks is a set locally diffeomorphic to the image of a map germ CBP : $\left(\mathbb{R}^{2}, 0\right) \rightarrow\left(\mathbb{R}^{3}, 0\right),(u, v) \mapsto$ $\left(3 \mathrm{u}^{4}-2 \mathrm{u}^{2} v^{2}, \mathrm{u}^{3}-\mathrm{u} v^{2}, v\right)$ and the 3-dimensional $\mathrm{D}_{4}^{+}$singularity is a set locally diffeomorphic to the image of a map germ $\mathrm{TD}^{+}:\left(\mathbb{R}^{2}, 0\right) \rightarrow\left(\mathbb{R}^{3}, 0\right),(u, v) \mapsto\left(u v, u^{2}+3 v^{2}, u^{2} v+v^{3}\right)$. Their pictures are shown in Figures $1-4$.

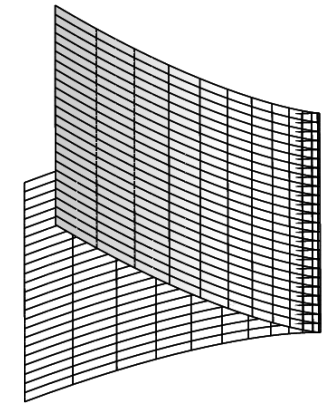

Figure 1: Cuspidal edge.

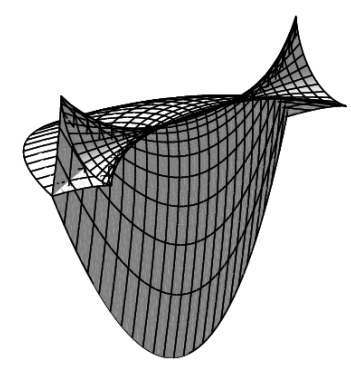

Figure 3: Cuspidal beaks.

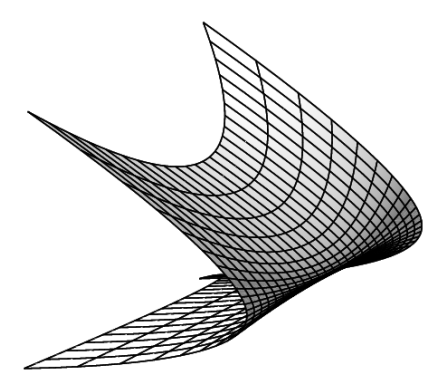

Figure 2: Cuspidal lips.

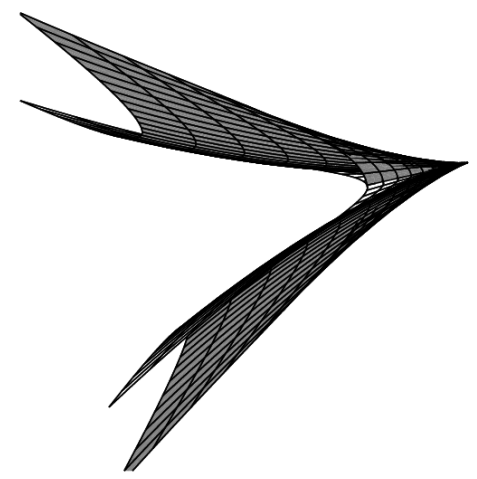

Figure 4: 3-dimensional $\mathrm{D}_{4}^{+}$singularity.

Proof. The assertion (1) is followed by claim (1) of Proposition 3.1 in this paper, and claim (1) is followed by Theorem 3.4 in [10]. The assertion (2) follows by claims (1) and (4) of Proposition 3.1 in this paper, and claim (2) follows by Theorem 3.5 in [10]. The assertion (3) follows by Proposition 3.2 in this paper, and claim (3) follows by Theorem 3.5 in [10].

\section{Examples}

As applications and illustration of the main results, we give two examples in this section.

Example 6.1. Let $\mathbf{X}(s, t)$ be a translation surface of $\mathbb{E}^{3}$ defined by

$$
\mathbf{X}(\mathrm{s}, \mathrm{t})=\left(\mathrm{s}, \mathrm{t}, \frac{1}{2} \mathrm{~s}^{2}-\frac{1}{2} \mathrm{t}^{2}\right)
$$


where $s \in[-0.2,0.2], t \in[-0.4,0.4]$. Let $f(s)=\frac{1}{2} s^{2}, g(t)=-\frac{1}{2} t^{2}$. It is easy to know that $f^{\prime}(0)=0, g^{\prime}(0)=0$, $f^{(3)}(0)=0, f^{(4)}(0)=0, k_{1}(0,0)=1>0, k_{2}(0,0)=-1<0$ and $k_{1}(0) k_{2}^{2}(0)\left(f^{(4)}(0)-3 k_{1}^{3}(0)\right)=-3<0$. By the claim (2) in Theorem 5.1, we know that the caustics of $C_{M_{1}}(s, t)$ are locally diffeomorphic to a cuspidal lips at $C_{M_{1}}(0,0)$. The pictures of this translation surface and one of its caustics are shown in Figure 5 . The pictures of the enlarged images of caustic, whose are locally diffeomorphic to a cuspidal lips are shown in Figure 6.

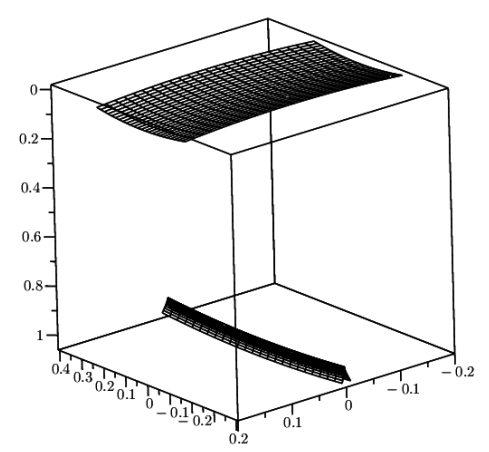

Figure 5: Translation surface and its caustic.

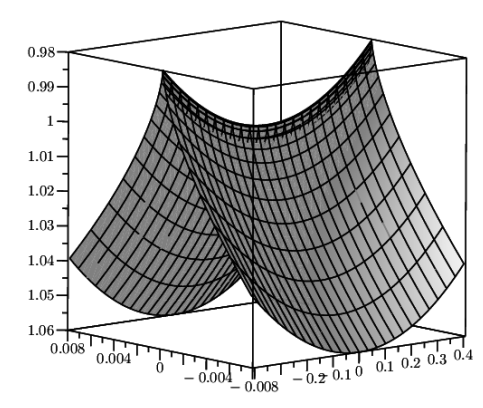

Figure 6: The enlarged image of caustic.

Example 6.2. Let $\mathbf{X}(s, t)$ be a translation surface of $\mathbb{E}^{3}$ defined by

$$
\mathbf{X}(\mathrm{s}, \mathrm{t})=(\mathrm{s}, \mathrm{t}, \cos (\mathrm{s})+\cos (\mathrm{t})-2),
$$

where $s \in[-0.2,0.2], t \in[-0.2,0.2]$. Let $f(s)=\cos (s)-1, g(t)=\cos (t)-1$. It is easy to know that $k_{1}(0)=k_{2}(0)=-1$ and $f^{\prime \prime}(0) g^{\prime \prime}(0)=1 \neq 0$. By the claim (3) in Theorem 5.1, we know that the caustics of $C_{M_{i}}(s, t)$ are locally diffeomorphic to a 3-dimensional $D_{4}^{+}$singularity at $C_{M_{i}}(0,0)$. The pictures of this translation surface and one of its caustics are shown in Figure 7. The pictures of the enlarged images of caustic, whose are locally diffeomorphic to a 3-dimensional $\mathrm{D}_{4}^{+}$singularity are shown in Figure 8.

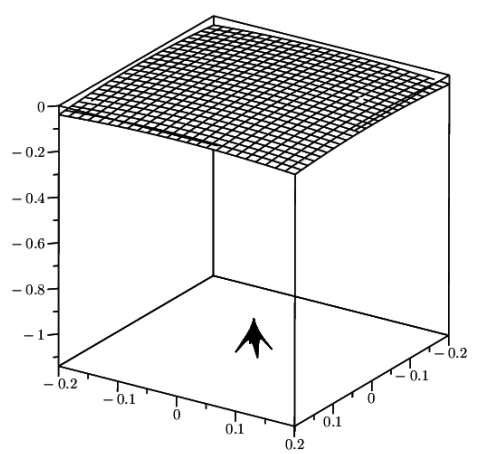

Figure 7: Translation surface and its caustic.

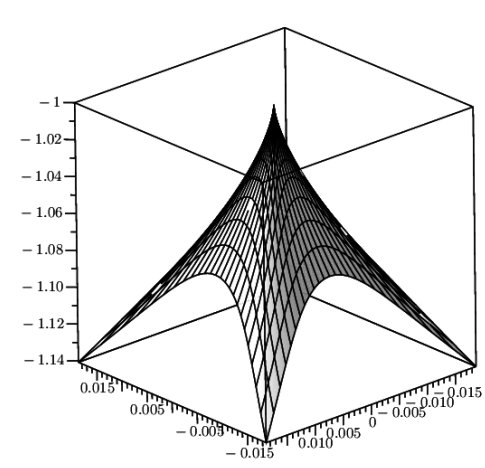

Figure 8: The enlarged image of caustic.

\section{Acknowledgment}

The second author was partially supported by the Project of Science and Technology of Heilongjiang Provincial Education Department of China No. UNPYSCT-2015103. The third author was partially supported by the Project of Science and Technology of Mudanjiang Government No. Z2016s0029. 


\section{References}

[1] J. A. Adam, The mathematical physics of rainbows and glories, Physics Reports, 356 (2002), 229-365. 1

[2] V. I. Arnold, Symplectic geometry and topology, J. Math. Phys., 41 (2000), 3307-3343. 1

[3] V. I. Arnol'd, S. M. Gueseřn-zade, A. N. Varchenko, Singularities of Differentiable Maps, Birkhäuser, Boston, (1986). 2

[4] M. Avendaño-Alejo, L. Castañeda, I. Moreno, Caustics and wavefronts by multiple reflections in a circular surface, Am. J. Physics., 78 (2010), 1195-1198. 1

[5] M. Avendaño-Alejo, D. González-Utrera, L. Castañeda, Caustics in a meridional plane produced by plano-convex conic lenses, J. Opt. Soc. Am. A, 28 (2011), 2619-2628. 1

[6] T. Banchoff, T. Gaffney, C. McCrory, Cusps of Gauss mappings, Pitman, Boston, (1982). 2, 2

[7] M. Bekkar, B. Senoussi, Translation surfaces in the 3-dimensional space satisfying $\Delta^{\mathrm{III}} \mathrm{r}_{\mathrm{i}}=\mu_{i} \mathrm{r}_{i}, \mathrm{~J}$. Geom., 103 (2012), 367-374. 1

[8] M. V. Berry, Disruption of images; the caustic touching theorem, J. Opt. Soc. Am. A, 4 (1987), 561-569. 1

[9] J. Ehlers, E. T. Newman, The theory of caustics and wave front singularities with physical applications, J. Math. Phys., 41 (2000), 3344-3378. 1

[10] T. Fukui, M. Hasegawa, Singularities of parallel surfaces, Tohoku Math. J., 64 (2012), 387-408. 2, 5

[11] W. Goemans, I. V. Woestyne, Translation Surfaces with Vanishing Second Gaussian Curvature in Euclidean and Minkowski 3-Space, Shaker Verlag, Aachen, (2007). 1

[12] W. Hasse, M. Kriele, V. Perlick, Caustics of wavefronts in general relativity, Classical Quantum Gravity, 13 (1996), 1161-1182. 1

[13] S. Izumiya, Differential Geometry from the viewpoint of Lagrangian or Legendrian singularity theory, World Scientific publishing Co., Singapore, (2007). 2

[14] L. Kong, D. Pei, On spacelike curves in hyperbolic space times sphere, Int. J. Geom. Methods Mod. Phys., 2014 (2014), 12 pages. 2

[15] H. Liu, Translation surfaces with dependent Gauss and mean curvature in 3-dimensional space, J. Neut., 14 (1993), 88-93. 1

[16] H. Liu, Translation surfaces with constant mean curvature in Euclidean 3-space, J. Geom., 64 (1999), 141-149.

[17] H. Liu, Y. Yu, Affine translation surfaces in 3-dimensional spaces, Proc. Japan Acad. Ser. A Math. Sci., 89 (2013), 111-113. 1

[18] R. López, M. I. Munteanu, Minimal translation surfaces in Sol S $^{3}$ J. Math. Soc. Japan, 64 (2012), 985-1003. 1

[19] I. R. Porteous, Geometric Differentiation for the Intelligence of Curves and Surfaces, Cambridge University Press, Cambridge, (1994). 2, 2, 3

[20] L. Verstraelen, J. Walrave, S. Yaprak, The minimal translation surfaces in Euclidean space, Soochow J. Math., 20 (1994), 77-82. 1

[21] J. Weiss, Bäcklund transformations, focal surfaces and the two-dimensional Toda lattice, Phys. Lett. A, 137 (1989), $365-368$. 1

[22] D. W. Yoon, On the Gauss map of translation surfaces in Minkowski 3-space, Taiwanese J. Math., 6 (2002), 389-398. 1

[23] Y. Yuan, H. L. Liu, Some new translation surfaces in 3-Minkowski space, J. Math. Res. Exposition, 31 (2011), 1123-1128. 1 